\title{
Surface micro-texturing design for improving tribological behaviour of graphene oxide thin films
}

\author{
XIAOLONG JIA ${ }^{1,2, *}$, CHONG WANG ${ }^{1}$, GUANGQI XIONG ${ }^{1}$ and FULIANG ZHU ${ }^{2}$ \\ ${ }^{1}$ College of Materials Science and Engineering, Chongqing University, Chongqing 400044, People's Republic of China \\ ${ }^{2}$ School of Material Science and Engineering, Lanzhou University of Technology, Lanzhou 730000, \\ People's Republic of China \\ *Author for correspondence (1415352949@qq.com)
}

MS received 18 December 2018; accepted 22 May 2019

\begin{abstract}
Effect of micro-scale surface texture with different densities on wear resistance of graphene oxide (GO) films was studied using a revolving ball-on-flat tribometer in humid environments. The micro-scale surface texture was produced by depositing about $363 \mathrm{~nm}$ thick GO films onto silicon substrates pre-textured with the patterns of dimples. The goal of the surface patterning was to improve the wear resistance of the GO films so that to extend lubricating life. The experimental results demonstrated excellent ability of the textured films to improve wear resistance of the silicon substrate. Furthermore, the GO films with an appropriate dimple area density (4\%) were effective in reducing the friction coefficient $(0.024)$ and exhibiting outstanding wear resistance owing to the entrapment of wear particles in the dimples. Finally, a simple friction mechanism model was drawn to explain the frictional properties of the different textured area density GO films.
\end{abstract}

Keywords. Graphene oxide films; micro-scale surface texture; appropriate textured area density; lower friction coefficient.

\section{Introduction}

Recently, micro high-tech devices including micro electromechanical system (MEMS), nano-electro mechanical system (NEMS) and disc storage system of ultrahigh density develop with an unsuspected speed [1,2]. However, with little driving force and high-specific surface area, the stability and lifespan of this kind of micro devices are shortened by its surface force dominated by adhesion and micro friction [2]. Meanwhile, many studies have confirmed that the thin solid lubricating film used in micro devices can effectively reduce the friction coefficient between the friction pairs, thereby reducing the wear rate and prolonging the service life [3-5].

As a new solid lubricating film, graphene manifests exceptionally high-mechanical properties and weak-interlay interaction, demonstrating excellent tribological properties [6-10]. Up to now, the preparation of industrial grade graphene films is still a great challenge. The graphene films can be prepared by the chemical vapour deposition method [11,12], oxidation-reduction modification [13], epitaxial growth method of crystal [14] and original method of micro mechanical cleavage [15]. However, the above preparation methods are harsh under experimental conditions, and most of them require high-experimental temperatures or energy states. In contrast, the electrophoretic deposition (EPD) is a simple and environmental friendly method of thin film preparation [16]. The basic principle of the EPD method is to deposit stable and uniform graphene oxide (GO) colloidal particles with negatively charged on a silicon wafer under the action of electric field, thereby rapidly depositing a high-quality GO film. The EPD method has the advantages of being deposited at room temperature, simple operation and a wide source of preparation materials. However, the film deposited in this method has problems, such as higher coefficient of friction and wearing, which largely limits the application in MEMS/NEMS.

On the other hand, laser texture technology has the advantages of fast processing speed, no pollution to the environment and controllable structure size, which makes it one of the surface processing technologies to obtain more applications [17]. Many studies have found that the laser surface texturing is an effective method to optimize material tribology performance which cannot only reduce the real contacting area between the two moving parts and result in decreasing the surface friction significantly, but also store the grinding debris in the micro-dimples to reduce the wearing prominently as well [18-21]. The synergistic lubrication effect of the laser surface texture method and solid lubrication film technology can achieve the purpose of reducing the friction coefficient and improving the anti-wear performance [20,21]. In addition, the carbon-based films of the texture have excellent function of anti-wear that is relevant to the special horizontal gradient structure and the stable change during the process of friction, too [22].

In this study, we use the indirect approach of laser texture to achieve the silicon texture by the density of $2,4,6$, and $10 \%$, respectively, and, then, produce thin GO films by the EPD method with the same voltage, $35 \mathrm{~V}$, which has been proved 
that high quality GO films can be prepared at this voltage [16]. Furthermore, it is showed, as a result, that the thin textured GO films lower the friction coefficient and improve the wearing resistance significantly.

\section{Experimental}

\subsection{Preparation of GO films}

In this paper, the GO films were prepared by the Hummers method. The obtained GO powder was dispersed in purified water and then ultrasonicated by an ultrasonic cleaner for $1-1.5 \mathrm{~h}$ to obtain a uniform and stable GO colloidal solution. Further details of the deposition process can be found in ref. [16]. Here, the EPD method is used to obtain GO films on $\mathrm{Si}(100)$ substrates. Prior to deposition, silicon wafers were cleaned in a piranha solution (a mixture of $98 \% \mathrm{H}_{2} \mathrm{SO}_{4}$ and $30 \% \mathrm{H}_{2} \mathrm{O}_{2}$ with a ratio of $\left.7: 3(\mathrm{v} / \mathrm{v})\right)$, subsequently washed with ultrapure water several times and blown dry with $\mathrm{N}_{2}$. The films were about $363 \mathrm{~nm}$ thick which are measured by using the cross-sectional scanning electron microscope (SEM). The deposition conditions are as follows: (1) applied dc negative voltage: $35 \mathrm{~V}$ and (2) deposition time kept at $3 \mathrm{~h}$. A schematic is presented in figure 1, which shows the GO films deposition process by the EPD method.

\subsection{Laser surface texturing of silicon substrate}

Micro-dimple patterns were created on the surfaces of the silicon substrates through a neodymium-yttrium-aluminium garnet laser with a wavelength of $1064 \mathrm{~nm}$, using a frequency of $10 \mathrm{kHz}$ and $90 \%$ overlapping rate of laser spot; the samples were processed with an average power of $8 \mathrm{~W}$ at a traverse speed of $4 \mathrm{~mm} \mathrm{~s}^{-1}$. After laser texturing, a gentle polishing process was used to remove bulges or burrs around the rim of the dimples. Four dimple area densities with the same dimple geometric parameters were designed. For all samples, the dimple diameter is $20 \mu \mathrm{m}$, the dimple depth is $13 \mu \mathrm{m}$ and ration depth/diameter is 0.65 . A schematic depiction of the deposition system on textured GO films, in this experiment, is presented in figure 2 .

\subsection{Characterization}

The thickness of samples was measured by cross-section SEM images (JEOL JSM-6701F). The nanostructures of films were observed by high-resolution transmission electron microscopy (HRTEM, JEOL 2010) operated at $200 \mathrm{kV}$. The sample films tested by HRTEM were deposited on the $\mathrm{NaCl}$ substrate, followed by the dissolution of the $\mathrm{NaCl}$ substrate with water. Micro-Raman backscattering spectra of the samples, deposited on the silicon substrate, were recorded on a LabRam HR800 spectrometer (HORIBA Jobin Yvon, France) operating with a $532 \mathrm{~nm}(2.3 \mathrm{eV})$ $\mathrm{Ar}$ laser as the excitation source. Laser power was taken carefully to avoid any possible heating damage on the a

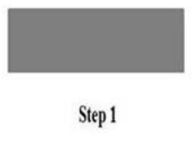

GO film thickness: $363 \mathrm{~nm}$

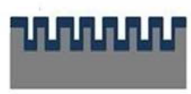

Step 3

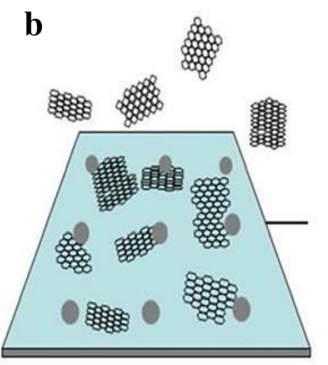

Figure 2. (a) Schematic of the preparation of specimens: step 1: silicon substrate. Step 2: laser surface texturing. Step 3: depositing GO films. (b) 3D stereogram of step 3 .

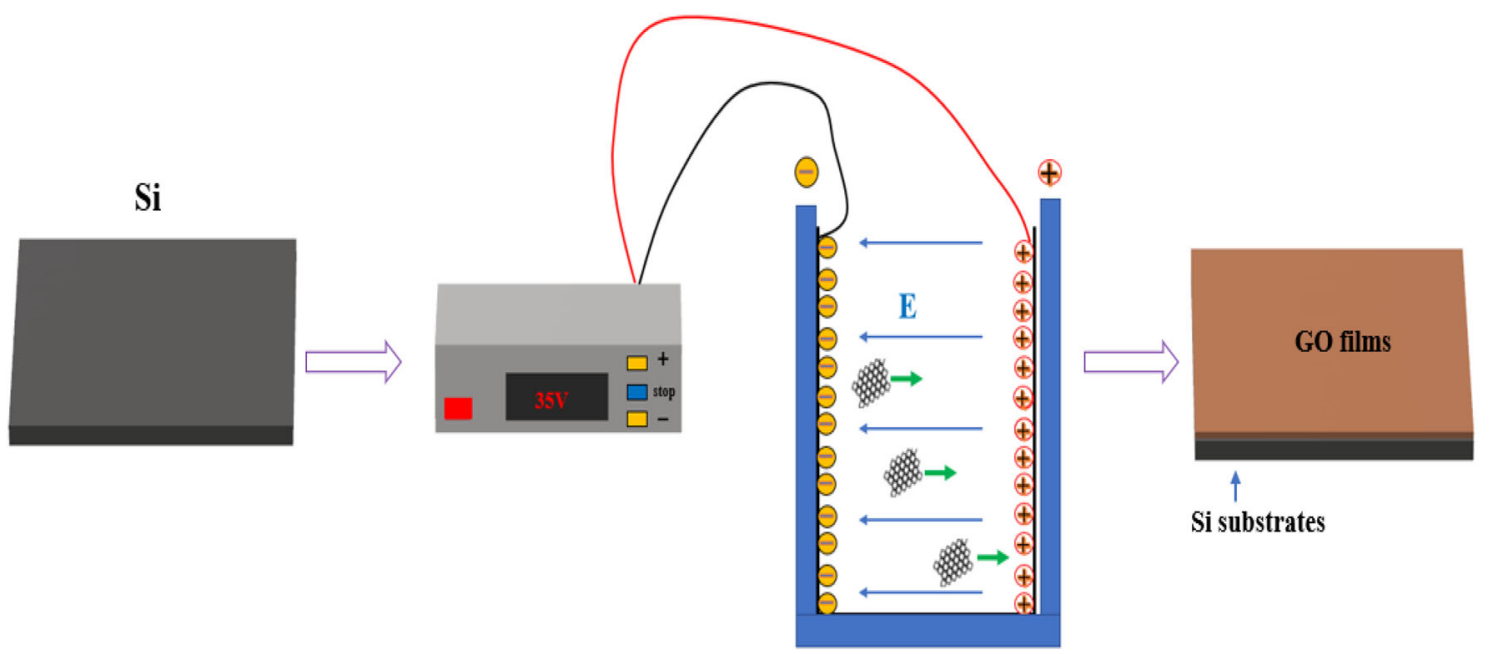

Figure 1. Schematic of depositing GO films by the EPD method. 


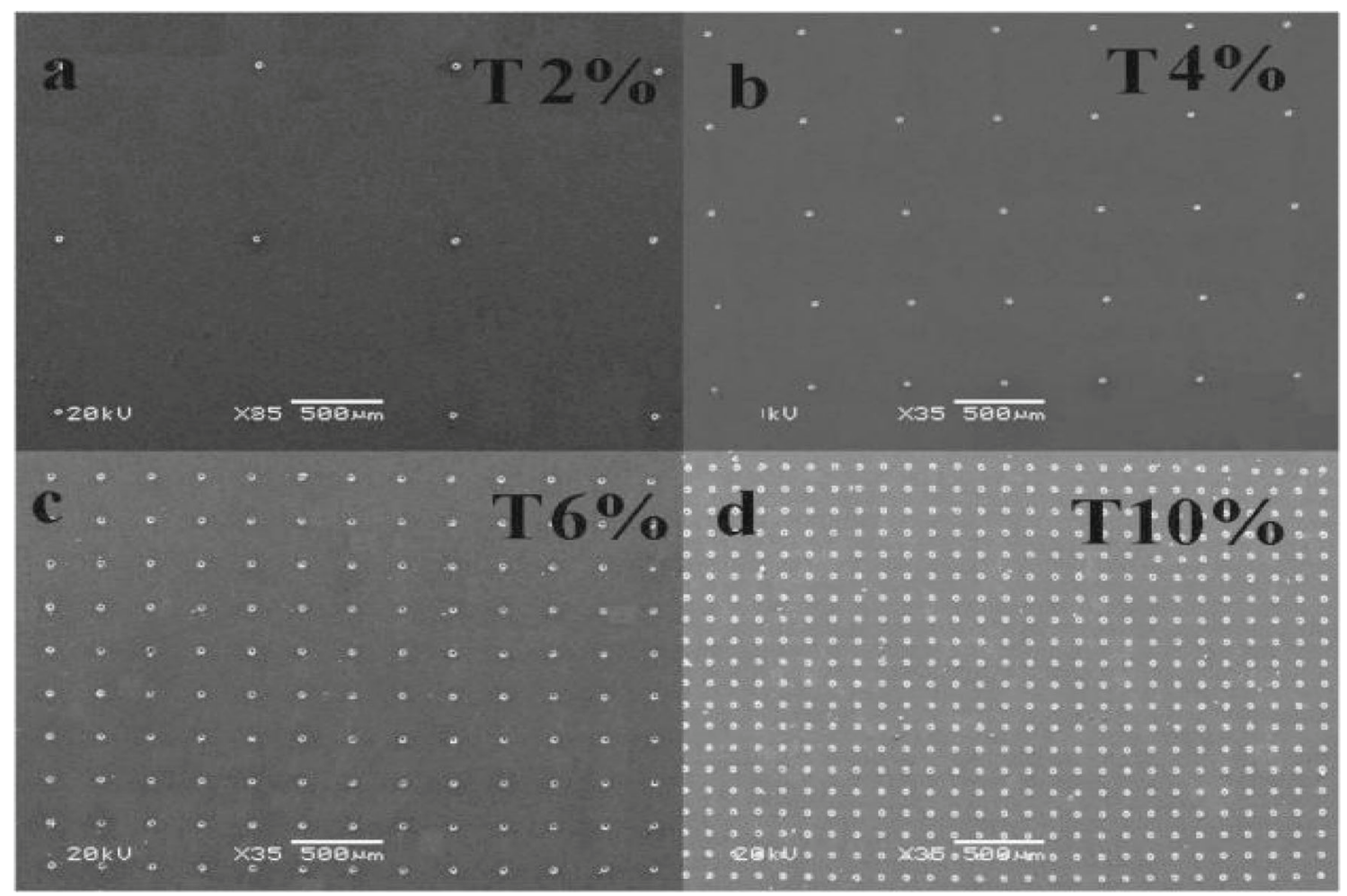

Figure 3. SEM images of the textured films with various textured area densities: (a) 2, (b) 4, (c) 6 and (d) $10 \%$.

samples. And the chemical states of the films were determined using a PHI-5702 multifunctional X-ray photoelectron spectrometer (Physical Electronics Inc, USA) (XPS, operating with $\mathrm{AlK} \alpha$ radiation and detecting chamber pressure of below 10-6 Pa), and the films were tested on the surface approach sensitivity as $40 \%$. The friction test was conducted using MS-T3000, which was a ball-on-plate revolving tribometer.

All tests were performed at room temperature and relative humidity ranging from 40 to $50 \%$ and the mating balls $(3 \mathrm{~mm}$, steel balls) were cleaned with acetone in an ultrasonic cleaner before each test. The rotation radius was $1 \mathrm{~cm}$, contact load was $100 \mathrm{mN}$ and an average rotation speed was $30 \mathrm{r} \mathrm{min}^{-1}$ within $30 \mathrm{~min}$. The corresponding wear scar morphologies of the samples were observed on a MAX3D three-dimensional surface profiler and the wear volume of the samples was calculated by measuring the cross-sectional area at three-different locations along the wear track.

\section{Result and discussion}

\subsection{Characterization of GO films}

SEM images of the four textured silicon surfaces with GO films are shown in figure 3. As can be seen from the image, figure $3 \mathrm{a}-\mathrm{d}$ depicts four different textured films with the area densities of 2, 4, 6 and 10\%, respectively. The area density defined as the ratio of the textured area to the whole area. Moreover, the micro-dimples were distributed uniformly on the silicon surface.

Figure $4 \mathrm{a}$ and $\mathrm{b}$ clearly shows the TEM and HRTEM images of films. As shown in figure $4 \mathrm{a}$, a typical image of layered graphene is overlapped and piled up together, while the well-stacked layered structure and ordered lattice fringes with $0.34 \mathrm{~nm}$ of graphene under high multiples are presented in figure $4 \mathrm{~b}$. To obtain further information on the microstructure of the as-prepared films, Raman spectroscopy is carried out. In figure $4 \mathrm{c}$, the Raman spectrum of the films exhibits the presence of $\mathrm{D}, \mathrm{G}$ and 2D bands. The $\mathrm{D}$ band at $1348 \mathrm{~cm}^{-1}$ arises from $\mathrm{sp}^{3}$ hybridized carbon [23], the peak at $1580 \mathrm{~cm}^{-1}$ arises from the $E_{2 \mathrm{~g}}$ zone centre mode of the crystalline graphite [24] and the 2D peak at about $2950 \mathrm{~cm}^{-1}$ represents the purity of the graphene [25], which agree with the Raman spectrum of GO films. Further, the ratio of ID/IG as 1.02 means less crystalline graphite in the films. The chemic structures of the as-prepared films are detected by XPS (figure 4d). We know from the survey of XPS spectra that the films contain the elements of carbon and oxide. We know from the XPS spectra of $\mathrm{Cls}$, the films containing $\mathrm{C}-\mathrm{O}$ (hydroxyl and epoxy groups $(287.1 \mathrm{eV})$ ) and carbonyl carbon $\mathrm{C}=\mathrm{O}(288.6 \mathrm{eV})$ are the main components except the carbon skeletons $\mathrm{C}-\mathrm{C}$ or $\mathrm{C}=\mathrm{C}$ $(284.8 \mathrm{eV})$ [26], which are further agreed with the GO sheet composition. As demonstrated above, the films with richlayered structure prepared by the EPD method are GO films. 

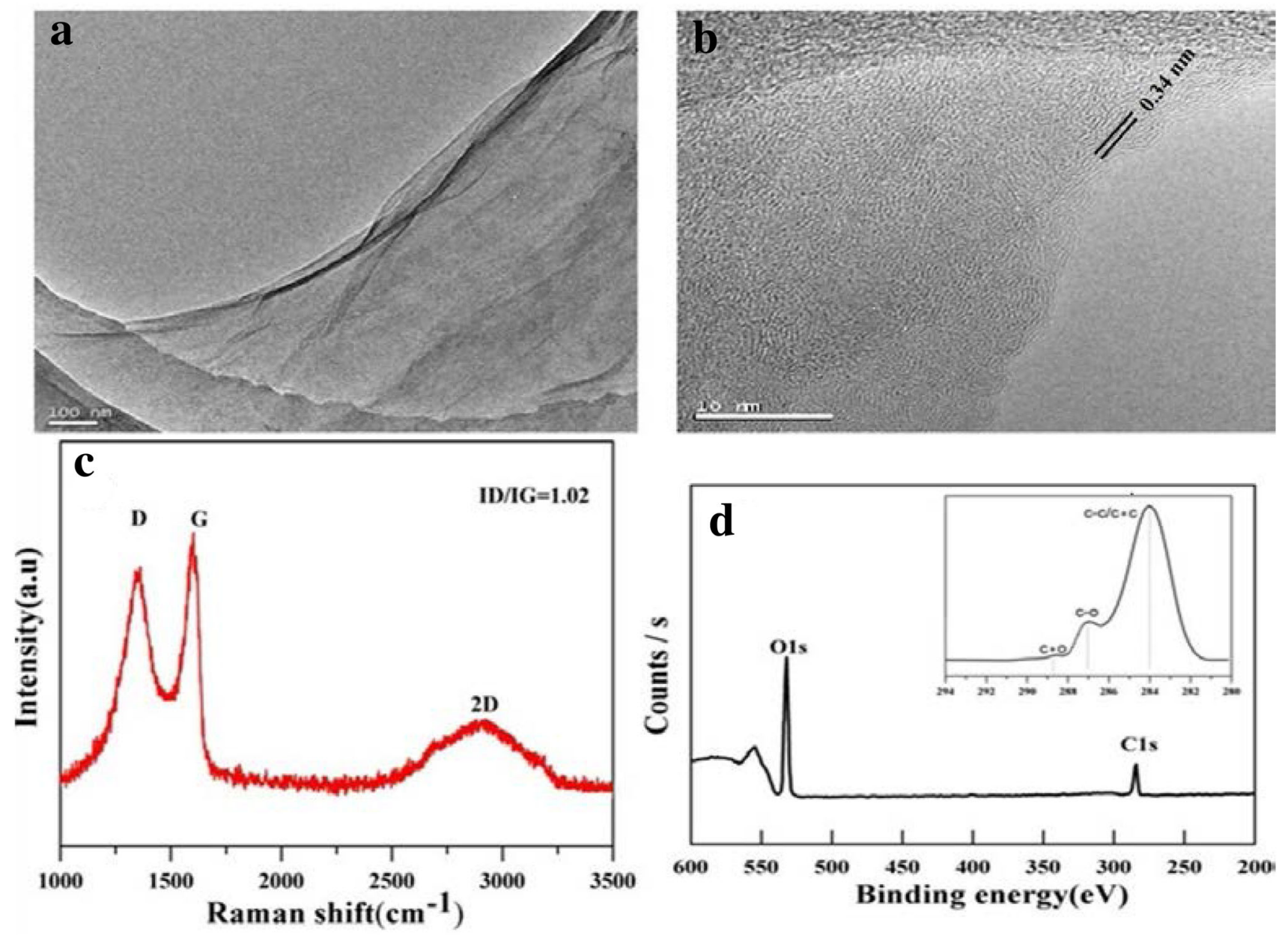

Figure 4. HRTEM images at (a) low and (b) high magnifications of GO films. (c) Typical Raman spectra and (d) XPS image of GO films.

\subsection{Friction and wear behaviour of the films}

Surface texturing reduces the friction coefficient obviously compared to the flat GO film in this experiment. All the experiments were repeated three times to ensure the repeatability of the experimental results. Figure 5 depicts the friction coefficient as a function of the sliding time for tests performed on flat GO, having textured area densities of 10, 6, 4 and $2 \%$ in humid air (relative humidity about $45 \%$ ), respectively. The friction coefficient value starts high on the flat GO, while decreases quickly during the run-in period and finally stops at a steady value of 0.095 (the friction coefficient of silicon is 0.4 ). On the other hand, the friction coefficient value starts low on the textured films, then, increases quickly and reaches a steady value after a shorter run-in period. The various performances of friction can be understood that flat GO films intensively restricted by surface adhesion, while textured films with micro-dimples are beneficial to eliminate surface adhesion. In particular, textured area density of $4 \%$ with the lowest friction coefficient (0.024), which only has about $1 / 4$ of the flat GO films, is observed.

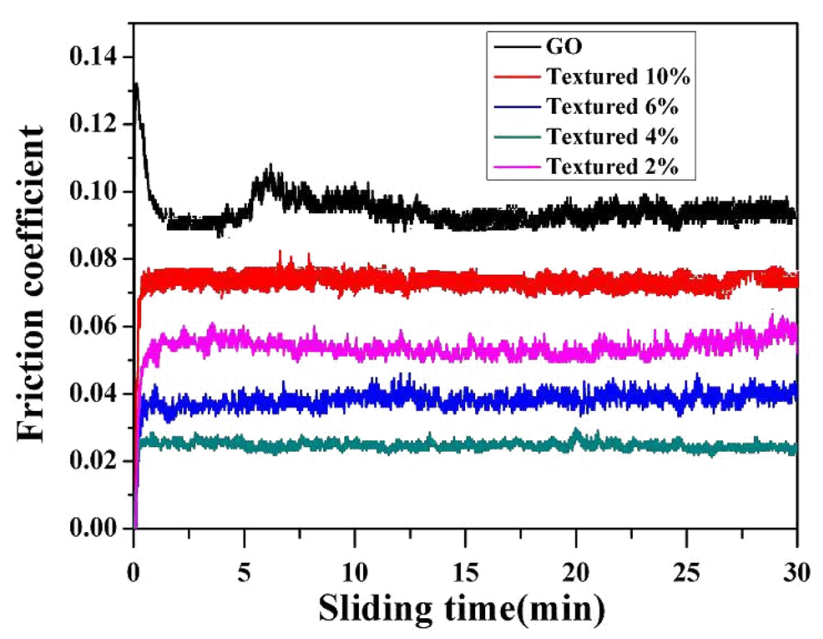

Figure 5. Friction coefficient curves with different films.

In addition, figure 6 indicates the Raman spectra of wear tracks on textured density of $10 \%$. Compared with the Raman spectra of the original film, the wear tracks' Raman spectra 
exists lower intensity of the D peak, and possesses stronger intensity of the $\mathrm{G}$ peak, which indicates that more longrange ordered graphene structures are formed in the film. The change of the structure is due to friction heat and energy undulation during the course of friction [27-29]. In detail, driven by repeated friction of steel ball, the GO film produces abundant friction heat [30], which causes the $\mathrm{sp}^{3}$ bond of oxygen containing functional groups (such as hydroxyl and carboxyl groups) on the edge of the GO film to break, and, the $\mathrm{sp}^{3}$ bond is transformed into a more stable and low-energy $\mathrm{sp}^{2}$ ordered graphene structure under the drive of the principle of minimum energy [21]. In other words, a subtle difference of the ID/IG value occurs in textured density of $10 \%$ which suffered the largest friction heat compared to original films, which means that the smaller change does not even occur in the other case. Thus, in this essay, the main influence on the tribological properties is still due to the difference in texture density, rather than the change in the microscopic phase structure of GO films before and after the friction heat drives it.

A simple friction mechanism model diagram was drawn to explain the frictional properties of the textured GO film. As shown in figure $7 \mathrm{a}$, for the texture density of $10 \%$, the grinding debris generated by the friction process cannot fill the

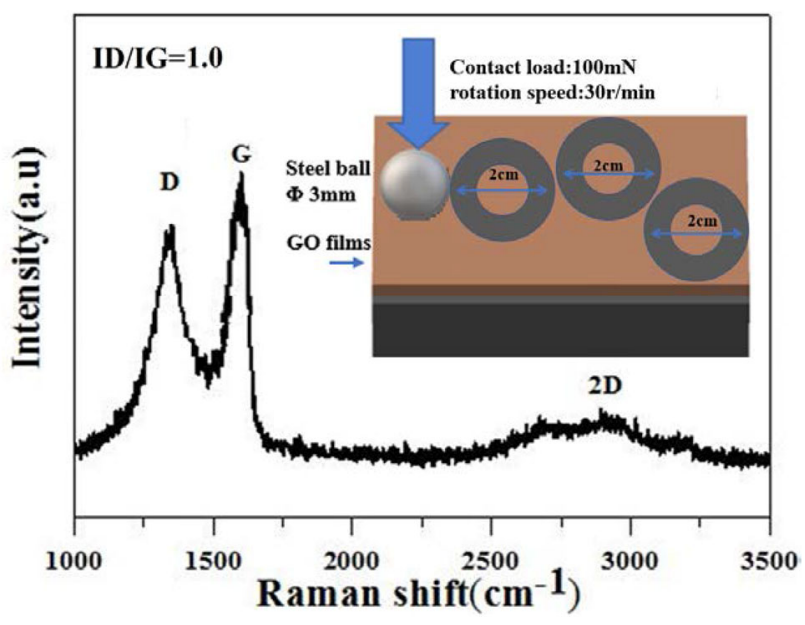

Figure 6. Raman spectra of wear track on textured density of $10 \%$, and the illustration at the upper right is schematic of friction experiment. micro-dimples, and the friction pair does not have a sufficient lubricating effect on the intermittent region, resulting in the friction coefficient of the textured density of $10 \%$ is higher than the texture densities of 6 and 4\%. In addition, as shown in figure $7 b$, the grinding debris of the GO film with a texture density of $4 \%$ is filled with micro-dimples during the friction process, which reduces the shear friction and provides sufficient solid lubrication. The synergistic effect of the laser surface texture method and solid lubrication technology effectively improves the tribological properties of the film [31,32].

However, for the texture density of $2 \%$, the grinding debris generated by the friction process cannot only fill the microdimples, but also distribute the remaining part on the contact surface to hinder the sliding of the steel ball (figure 7c), which leads to higher friction coefficient compared with the texture density of $4 \%$. All the above explanations show that the textured GO film deposited by the EPD method exhibits the best tribological properties with an optimum texture density. This phenomenon has also been confirmed by some literature studies. Pettersson et al [33] studied the effect of the texture size on the tribological properties of diamond-like carbon (DLC) films deposited on silicon-based surface texture under boundary lubrication conditions. The results show that the suitable texture size can improve the friction and wear properties. Ding et al [22] studied the tribological properties of DLC films with different texture densities. The results show that DLC films exhibit the best tribological properties at the optimum texture density.

The importance of using surface-texturing in establishing the excellent anti-wear behaviour is demonstrated in figure 8 . The wear volume of samples is calculated by measuring the cross-sectional area at three-different locations along the wear track. To obtain more accurate data, the results were repeatedly tested for more than three times. As we can see from the histogram of the wear volume, the friction coefficients and the wear volume of the films changed in the same tendency. In particular, the lowest wear volume $\left(2.3 \times 10^{-14} \mathrm{~m}^{3}\right)$ can be obtained for the textured density of $4 \%$ film and $1 / 2$ to flat GO films. In addition, the inset of histogram is wear tracks of the textured density of $6 \%$. As shown in the inset, micro-dimples of films covered by wear debris for a short sliding time. The wear debris stored by
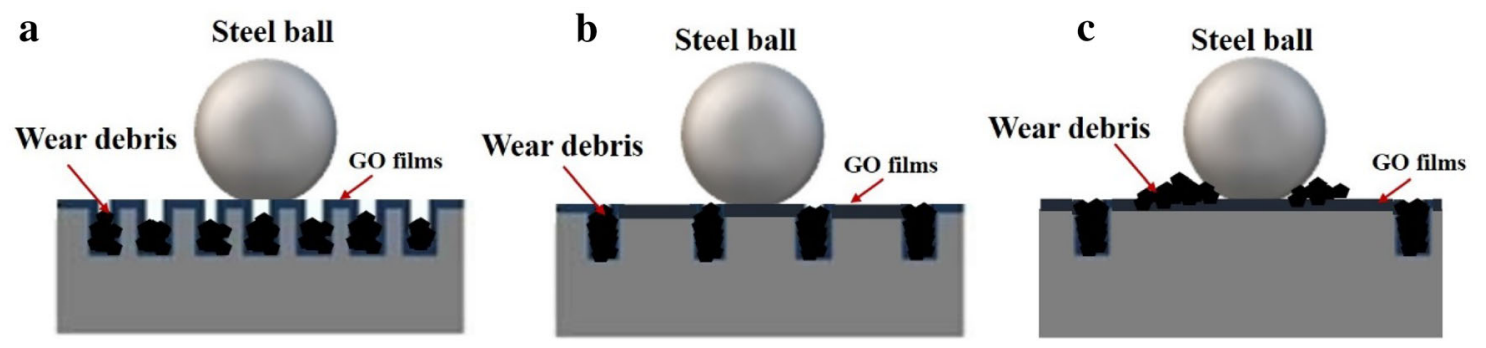

Figure 7. Friction mechanism model of GO films with different texture densities: (a) 10, (b) 4 and (c) $2 \%$. 


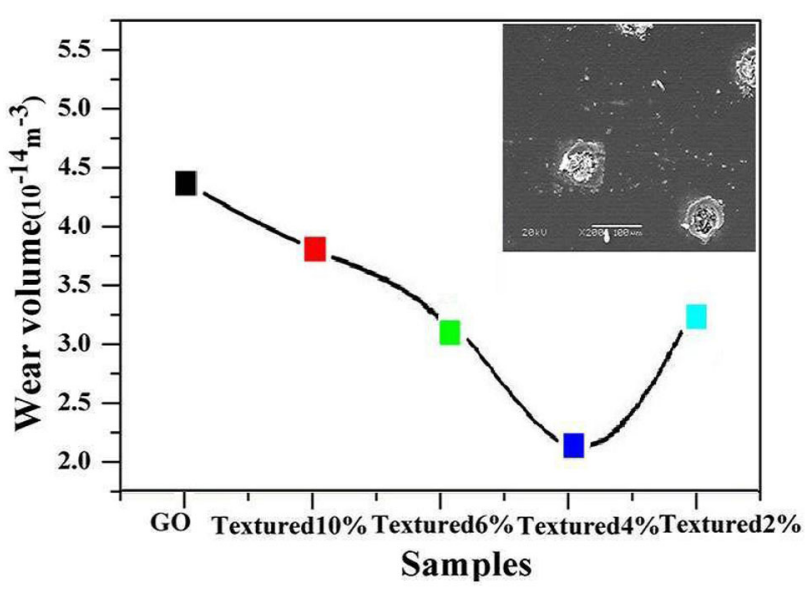

Figure 8. Wear volume curve of samples. Inset shows the typical wear tracks of the textured film.

micro-dimples can play a key role in secondary lubrication by reducing the wear rate of films significantly $[34,35]$.

\section{Conclusions}

From the results presented in this paper, the following conclusions can be drawn:

(a) The tribological properties of GO films on flat and textured silicon substrates with different micro-dimple area densities were investigated. Compared with the flat GO film, the surface textured film not only showed lower friction coefficient, but also exhibited lower wear volume, which attributed to the synergistic lubrication effect of both micro-textured structural designing and solid lubrication films.

(b) The texturing density area of $4 \%$ exhibited the lowest friction coefficient $(0.024)$ and wear volume $(2.3 \times$ $10^{-14} \mathrm{~m}^{3}$ ), the wear rate is $1 / 2$ of flat GO films. In addition, the Raman spectra of wear tracks on the textured density of $10 \%$ showed that the GO film is very stable during the friction process, and almost no structural transformation occurs, which indicated that the tribological properties of the GO film were only affected by texture density in this paper.

(c) A simple model was proposed to effectively explain the frictional properties of GO films with different texture densities. For the texture density of $10 \%$, grinding debris generated during the friction process is less and cannot fill the micro-dimples, the synergistic lubrication effect is not obvious, resulting in a high-friction coefficient; for the texture density of $2 \%$, grinding debris generated by the friction process cannot only fill the micro-dimples, but also distribute the remaining part on the contact surface to hinder the sliding of the steel ball, which leads to higher friction coefficient compared with the texture density of $4 \%$. However, the grinding debris of the GO film with a texture density of $4 \%$ is filled with micro-dimples completely during the friction process, which reduces the shear friction and provides sufficient solid lubrication. The synergistic effect of micro-textured structural designing and solid lubrication films effectively improves the tribological properties of the film.

\section{Acknowledgements}

We are grateful to the National Natural Science Foundation of China (grant nos. 51275508, 51205383) for the financial support.

\section{References}

[1] Bishop D, Pardo F, Bolle C and Giles R 2012 J. Low. Temp. Phys. 169386

[2] Palacio M and Bhushan B 2008 Adv. Mater. 201194

[3] Wang Q, Wang C B, Zhang J Y and He D Y 2007 Appl. Phys. Lett. 91141902

[4] Wang C B, Yang S R, Wang Q and Zhang J Y 2008 Nanotechnology 19225709

[5] Meng Y S, Jia X L, Zhu F L and Zhang J Y 2017 Bull. Mater. Sci. 401029

[6] Ou J F, Wang Y, Wang J Q, Liu S, Li Z P and Yang S R 2011 J. Phys. Chem. 11510080

[7] Liu H, Li Y Q, Wang T M and Wang Q H 2012 J. Mater. Sci. 47 1867

[8] Tai Z X, Chen Y F, An Y F, Yan X B and Xue Q J 2012 Tribol. Lett. 4655

[9] Liu Z H, Shu D, Li P F and Cheng X H 2014 RSC Adv. 415937

[10] Huang T, Li T S, Xin Y S, Jin B C, Chen Z X, Su C et al 2014 RSC Adv. 419814

[11] Srivastava S K, Shukla A K, Vankar V D and Kumar V 2005 Thin Solid Films 492124

[12] Zhu M Y, Wang J J, Holloway B C and Outlaw R A 2007 Carbon 452229

[13] Hummers W and Offeman R 1958 J. Am. Chem. Soc. 801339

[14] Sutter P W, Flege J I and Sutter E A 2008 Nat. Mater. 7406

[15] Geim A K and Novoselov K S 2007 Nat. Mater. 6183

[16] Liang H Y, Bu Y F, Zhang J Y, Cao Z Y and Liang A M 2013 ACS Appl. Mater. Interfaces 56369

[17] Ibatan T, Uddin M and Chowdhury M 2015 Surf. Coat. Tech. 272102

[18] Robertson J. 2002 Mater. Sci. Eng. R 37129

[19] Etsion I 2003 Tribol. Lett. 17733

[20] Wang X L, Kato K, Adachi K and Aizawa K 2003 Tribol. Int. 36 189

[21] Dumitru G, Romano V, Weber H P, Pimenov S, Kononenko T, Hermann J et al 2003 Diam. Relat. Mater. 121034

[22] Ding Q, Wang L P, Wang Y X, Wang S C, Hu L T and Xue Q J 2011 Tribol. Lett. 41439

[23] Bosch-Navarro C, Coronado E, Marti-Gastaldo C, SanchezRoyo J F and Gomez M 2012 Nanoscale 43977

[24] Oscar A, Vargas C, Alvaro C and Julian M 2012 Nanoscale 4 2083 
[25] Zhu X L, Wang W H and Yan W 2012 Phys. Rev. B 86 241301

[26] Su C Y, Xu Y P, Zhang W J, Zhao J W, Tang X H, Tsai C H et al 2009 Chem. Mater. 215674

[27] Liu Y, Erdemir A and Meletis E I 1996 Surf. Coat. Tech. 8248

[28] Wang Y F, Gao K X, Zhang B, Wang Q and Zhang J Y 2018 Carbon 13749

[29] Gong Z B, Shi J, Zhang B and Zhang J Y 2017 Carbon 116 310
[30] Ferrari A C and Robertson J 2000 Phys. Rev. B 6114095

[31] Pettersson U and Jacobson S 2003 Tribol. Int. 36857

[32] He D Q, Zheng S X, Pu J B, Zhang G A and Hu L T 2015 Tribol. Int. 8220

[33] Pettersson U and Jacobson S 2004 Tribol. Lett. 17553

[34] Zhang B, Huang W, Wang J and Wang X 2013 Tribol. Int. 65 138

[35] Kustandi T S, Choo J H, Low H Y and Sinha S K 2010 J. Phys. D 43015301 